\title{
CONSIDERACIONES BIOÉTICAS SOBRE LA RELACIÓN MÉDICO- PACIENTE PARA EL USO DEL CANNABIS MEDICINAL EN PERÚ
}

\author{
Sarah Carracedo ${ }^{1, a}$
}

\begin{abstract}
RESUMEN
En noviembre de 2017 se despenalizó en el Perú el uso medicinal del cannabis. Los desafíos que ello plantea son diversos y el presente artículo se centra en los relativos a la relación médico-paciente. Dado el status quo del cannabis medicinal, es necesario que el protocolo de tratamiento médico que el Ministerio de Salud debe elaborar, formule condiciones claras que guíen la decisión de los médicos de prescribir cannabis sin que ello afecte sus obligaciones éticas de promover el bien y de no causar daño a sus pacientes. Este artículo desarrolla tres consideraciones importantes para la prescripción de cannabis medicinal. En primer lugar, recomienda el registro y educación de los médicos que deciden prescribir cannabis medicinal. En segundo lugar, propone criterios generales que indican los casos en los que el uso del cannabis medicinal es apropiado, y finalmente, establece la necesidad de que exista una relación médico-paciente bona-fide para la prescripción del cannabis medicinal.
\end{abstract}

Palabras clave: Marihuana Medicinal; Cannabis; Relaciones Médico-Paciente; Bioética. (Fuente: DeCS BIREME).

\section{BIOETHICAL CONSIDERATIONS IN THE PHYSICIAN-PATIENT RELATIONSHIP FOR THE USE OF MEDICINAL CANNABIS IN PERU}

\begin{abstract}
In November 2017, medical use of cannabis was decriminalized in Peru. The challenges are diverse and this article focuses on those challenges related to the doctor-patient relationship. Given the status quo of medicinal cannabis, the medical treatment protocol to be developed by the Ministry of Health must formulate clear conditions to guide the physicians' decision to prescribe cannabis without affecting their ethical obligations to promote good and cause no harm to their patients. This article develops three important considerations for prescribing medicinal cannabis. First, it recommends the registration and education of physicians who decide to prescribe medicinal cannabis. Secondly, it proposes general criteria specifying the cases in which the use of medicinal cannabis is appropriate and, finally, it establishes the need for a bona-fide doctor-patient relationship for the prescription of medicinal cannabis.
\end{abstract}

Keywords: Medical Marijuana, Cannabis, Medical-patient relationship, Bioethics. (source: MeSH NLM).

\section{INTRODUCCIÓN}

El uso medicinal de la marihuana es un tema controversial en diversas sociedades pues distintas cuestiones científicas, legalesyéticas estáninvolucradas.Apesardeello, actualmente existe una tendencia a favor de su despenalización. En el 2001, Canadá se convirtió en el primer país del mundo en autorizar la marihuana medicinal, y desde entonces, otros países han seguido el mismo camino: Holanda (2003), Italia (2007), Colombia (2015), Australia (2016), Puerto Rico (2017), 29 estados de los Estados Unidos, entre otros ${ }^{(1)}$. En el 2017, con la Ley $N^{\circ} 30681$, Ley que regula el uso medicinal y terapéutico del cannabis y sus derivados (2), Perú se sumó a la lista de países que han optado por garantizar el acceso al cannabis medicinal en el nombre del derecho a la salud.

La aprobación del uso del cannabis con fines medicinales alrededor del mundo no ha sido uniforme. Las regulaciones son diferentes (en algunos países, las leyes son más restrictivas que en otros) y han sido el resultado de pronunciamientos de cortes constitucionales, de referéndums 0 de procesos legislativos (Tabla 1). A pesar de ello, las preocupaciones en torno a la despenalización del cannabis medicinal suelen ser

Pontifica Universidad Católica del Perú

Abogada, Magíster en Bioética

Recibido: 13/12/2018 Aprobado: 17/04/2019 En línea: 28/06/2019

Citar como: Carracedo S. Consideraciones bioéticas en la relación médico-paciente para el uso del cannabis medicinal en Perú. Rev Peru Med Exp Salud Publica. 2019;36(2):334-40. doi: http://dx.doi.org/10.17843/rpmesp.2019.362.3293. 
Tabla 1. Cuadro comparativo de regulaciones del cannabis medicinal

\begin{tabular}{|c|c|c|c|c|c|c|}
\hline País & $\begin{array}{c}\text { Año de } \\
\text { despenalización } \\
\text { del uso del } \\
\text { cannabis } \\
\text { medicinal }\end{array}$ & $\begin{array}{l}\text { Regulación } \\
\text { vigente }\end{array}$ & $\begin{array}{l}\text { Requisitos para el } \\
\text { paciente }\end{array}$ & $\begin{array}{l}\text { Requisitos para el } \\
\text { médico }\end{array}$ & $\begin{array}{l}\text { Tipo y cantidad de } \\
\text { Cannabis }\end{array}$ & $\begin{array}{l}\text { Indicaciones } \\
\text { terapéuticas }\end{array}$ \\
\hline $\begin{array}{l}\text { EEUU- } \\
\text { Califormia }\end{array}$ & 1996 & $\begin{array}{l}\text { Ley de seguridad } \\
\text { para el uso adulto y } \\
\text { medicinal del cannabis } \\
\text { (Medicinal and } \\
\text { Adult-Use Cannabis } \\
\text { Regulatory Safety Act- } \\
\text { MAUCRSA), 2017 }\end{array}$ & $\begin{array}{l}\text { El paciente debe contar } \\
\text { con: (1) la recomendación } \\
\text { del médico tratante; } \\
\text { y (2) una tarjeta de } \\
\text { identificación de paciente } \\
\text { calificado para usar el } \\
\text { cannabis medicinal. } \\
\text { (3) El Cannabis } \\
\text { puede adquirirse en } \\
\text { dispensadoras licenciadas } \\
\text { o auto cultivarse. }\end{array}$ & $\begin{array}{l}\text { Para ser médico tratante } \\
\text { se requiere: (1) poseer } \\
\text { una licencia para ejercer } \\
\text { la medicina, podiatria u } \\
\text { osteopatía emitida por la } \\
\text { Junta Médica de Califormia, } \\
\text { la Junta de Medicina } \\
\text { Podiátrica de California o la } \\
\text { Junta Médica Osteopática } \\
\text { de Califormia; (2) haber } \\
\text { asumido la responsabilidad } \\
\text { de la atención médica, el } \\
\text { tratamiento, el diagnóstico, } \\
\text { el asesoramiento o la } \\
\text { derivación del paciente; } \\
\text { y (3) haber realizado } \\
\text { un examen médico } \\
\text { del paciente antes de } \\
\text { determinar la existencia } \\
\text { de una condición médica } \\
\text { seria yel uso del cannabis } \\
\text { medicinal. }\end{array}$ & $\begin{array}{l}\text { (1) Un paciente calificado } \\
\text { puede poseer un máximo } \\
\text { de } 8 \text { onzas de cannabis } \\
\text { seco (dried cannabis). } \\
\text { Adicionalmente, cada } \\
\text { paciente puede tener } \\
\text { un máximo de } 6 \text { plantas } \\
\text { de cannabis maduras o } \\
12 \text { plantas de cannabis } \\
\text { inmaduras. (2) Si el médico } \\
\text { tratante recomienda } \\
\text { cantidades que exceden } \\
\text { las permitidas, el paciente } \\
\text { puede poseer la cantidad } \\
\text { requerida para sus } \\
\text { necesidades autorizada por } \\
\text { el médico. }\end{array}$ & $\begin{array}{l}\text { El cannabis medicinal } \\
\text { puede recomendarse } \\
\text { para condiciones médicas } \\
\text { serias. Estas condiciones } \\
\text { son: (1) Sindrome de } \\
\text { Inmunodeficiencia Adquirida } \\
\text { (SIDA); (2) Anorexia; } \\
\text { (3) Artritis; (4) Cachexia; } \\
\text { (5) Cáncer; (6) Dolor } \\
\text { crónico; (7) Glaucoma; } \\
\text { (8) Migrañas; (9) Espasmos } \\
\text { musculares persistentes } \\
\text { como los espasmos } \\
\text { asociados a la esclerosis } \\
\text { múltiple; (10) Convulsiones } \\
\text { como las asociadas a la } \\
\text { epilepsia. (11) Náuseas } \\
\text { severas (12) Cualquier otro } \\
\text { sintoma médico crónico o } \\
\text { persistente que: (a) Limita } \\
\text { sustancialmente la habilidad } \\
\text { de la persona de llevar a } \\
\text { cabo una o más actividades } \\
\text { importantes de la vida según } \\
\text { lo define la Ley Federal } \\
\text { de Estadounidenses con } \\
\text { Discapacidades de 1990; } \\
\text { o (b) Si no se alivia, puede } \\
\text { causar graves daños a la } \\
\text { seguridad o la salud física ylo } \\
\text { mental del paciente. }\end{array}$ \\
\hline Canadá & 2001 & $\begin{array}{l}\text { Ley de Cannabis } \\
\text { (Cannabis Act), } 2018\end{array}$ & $\begin{array}{l}\text { (1) Pacientes autorizados } \\
\text { por profesionales de } \\
\text { la salud (médicos } \\
\text { o enfermeras). (2) } \\
\text { EI Cannabis puede } \\
\text { adquirirse directamente } \\
\text { de un vendedor con } \\
\text { licencia federal; o (3) } \\
\text { puede producirse en } \\
\text { cantidades limitadas por el } \\
\text { paciente que cuente con } \\
\text { registro para ello; o (4) } \\
\text { puede ser producido por } \\
\text { otra persona designada } \\
\text { por el paciente. }\end{array}$ & $\begin{array}{l}\text { El médico o enfermera } \\
\text { debe: (1) practicar la } \\
\text { medicina o enfermeria; } \\
\text { y (2) no estar restringido } \\
\text { a autorizar el uso de } \\
\text { cannabis según las } \\
\text { leyes de la provincia. } \\
\text { Estos profesionales } \\
\text { también pueden poseer } \\
\text { marihuana fresca o seca } \\
\text { (fresh or dried marijuana) } \\
\text { o aceite de cannabis, } \\
\text { transferir ylo administrar } \\
\text { la sustancia al paciente. } \\
\text { Para la prescripción del } \\
\text { cannabis debe emitirse } \\
\text { un documento médico en } \\
\text { el cual debe, entre otros, } \\
\text { indicarse la cantidad diaria } \\
\text { autorizada expresada en } \\
\text { gramos de marihuana } \\
\text { seca. }\end{array}$ & $\begin{array}{l}\text { No existen límites en } \\
\text { la posesión privada de } \\
\text { cannabis con fines médicos; } \\
\text { aunque las cantidades de } \\
\text { posesión pública sí son } \\
\text { restringidas. }\end{array}$ & $\begin{array}{l}\text { La norma federal no establece } \\
\text { condiciones médicas que } \\
\text { habilitan el uso del cannabis } \\
\text { medicinal; aunque a nivel } \\
\text { provincial suelen existir pautas } \\
\text { de los colegios profesionales } \\
\text { que determinan ello. }\end{array}$ \\
\hline Holanda & 2002 & $\begin{array}{l}\text { Convención Única } \\
\text { de } 1981 \text { sobre } \\
\text { estupefacientes } \\
\text { (articulo 23); Ley del } \\
\text { opio (Opium Act) y } \\
\text { los Lineamientos } \\
\text { de la política de } \\
\text { exenciones a la Ley } \\
\text { del Opio (Policy } \\
\text { Guidelines Opium Act } \\
\text { Exemptions), } 2002\end{array}$ & $\begin{array}{l}\text { (1) Pacientes autorizados } \\
\text { por prescripción médica. } \\
\text { (2) El Cannabis puede } \\
\text { adquirise en cualquier } \\
\text { farmacia holandesa. }\end{array}$ & $\begin{array}{l}\text { (1) Médico habilitado } \\
\text { para ejercer medicina. } \\
\text { (2) El médico solo puede } \\
\text { prescribir cannabis si el } \\
\text { paciente ha intentado otros } \\
\text { tratamientos sin éxito. (3) } \\
\text { El médico debe indicar la } \\
\text { dosis y tipo de cannabis } \\
\text { medicinal en la receta } \\
\text { médica. }\end{array}$ & $\begin{array}{l}\text { Existen } 5 \text { variedades } \\
\text { farmacéuticas de } \\
\text { cannabis con composición } \\
\text { cannabinoide estandarizada: } \\
\text { (1) Bedrobino(囚; (2) } \\
\text { Bedrocan®; (3) Bedica®; } \\
\text { (4) Bediol(8) (5) Bedrolite®. } \\
\text { La oficina del Cannabis } \\
\text { Medicinal (Office of } \\
\text { Medicinal Cannabis - OMC) } \\
\text { publica guías en tomo } \\
\text { a las dosis y vías de } \\
\text { administración. }\end{array}$ & $\begin{array}{l}\text { La norma no establece } \\
\text { condiciones médicas que } \\
\text { habilitan el uso del cannabis } \\
\text { medicinal; pero la OMC } \\
\text { publica guías en torno a las } \\
\text { indicaciones terapéuticas, } \\
\text { sin restringir el juicio de los } \\
\text { médicos. }\end{array}$ \\
\hline
\end{tabular}


Tabla 1. Cuadro comparativo de regulaciones del cannabis medicinal (Viene de la página 335)

\begin{tabular}{|c|c|c|c|c|c|c|}
\hline País & $\begin{array}{c}\text { Año de } \\
\text { despenalización } \\
\text { del uso del } \\
\text { cannabis } \\
\text { medicinal }\end{array}$ & $\begin{array}{l}\text { Regulación } \\
\text { vigente }\end{array}$ & $\begin{array}{l}\text { Requisitos para } \\
\text { el paciente }\end{array}$ & $\begin{array}{l}\text { Requisitos para el } \\
\text { médico }\end{array}$ & $\begin{array}{c}\text { Tipo y cantidad de } \\
\text { Cannabis }\end{array}$ & Indicaciones terapéuticas \\
\hline $\begin{array}{l}\text { Puerto } \\
\text { Rico }\end{array}$ & 2017 & $\begin{array}{l}\text { Ley para manejar el } \\
\text { Estudio, Desarrollo } \\
\text { e Investigación del } \\
\text { Cannabis para la } \\
\text { Innovación, Normas } \\
\text { Aplicables y Límites } \\
\text { (“Ley Medicinal"), } 2017 \\
\text { y Reglamento N } \\
9038,2018\end{array}$ & $\begin{array}{l}\text { El paciente debe } \\
\text { contar con: (1) La } \\
\text { recomendación para } \\
\text { el cannabis medicinal } \\
\text { de un médico } \\
\text { autorizado y (2) el } \\
\text { registro ante la Junta } \\
\text { Reglamentadora } \\
\text { de Cannabis } \\
\text { Medicinal. (3) Solo } \\
\text { puede comprarse } \\
\text { Cannabis Medicinal } \\
\text { en un dispensario } \\
\text { debidamente } \\
\text { licenciado. }\end{array}$ & $\begin{array}{l}\text { (1) Médico licenciado en Puerto } \\
\text { Rico para ejercer la medicina. } \\
\text { (2) Debe estar registrado para } \\
\text { recomendar cannabis medicinal, } \\
\text { cumpliendo los siguientes } \\
\text { requisitos: (A) Certificado de } \\
\text { adiestramiento inicial de } 6 \\
\text { horas crédito ofrecido por la } \\
\text { Oficina de Cannabis Medicinal } \\
\text { u otro proveedor autorizado; } \\
\text { (B) contar con licencia vigente } \\
\text { para prescribir drogas; (C) } \\
\text { Certificado de buena conducta } \\
\text { expedido por la Junta de } \\
\text { Licenciamiento y Disciplina } \\
\text { Médica del Departamento de } \\
\text { Salud. (3) El médico autorizado } \\
\text { debe cumplir además con un } \\
\text { mínimo de seis horas crédito } \\
\text { de educación continua anuales } \\
\text { por cada periodo de } 3 \text { años. (4) } \\
\text { La recomendación del uso del } \\
\text { cannabis medicinal debe darse } \\
\text { en el marco de una relación } \\
\text { médico-paciente bona fide. }\end{array}$ & $\begin{array}{l}\text { (1) Se considera cannabis } \\
\text { medicinal a todo compuesto, } \\
\text { producto, derivado, mezcla } \\
\text { o preparación de todas las } \\
\text { partes de la planta Cannabis } \\
\text { Sativa y Cannabis Indica y } \\
\text { cualquier hibrido de estas, } \\
\text { de sus semillas, de su flor o } \\
\text { de su resina. No incluye los } \\
\text { tallos maduros ni las fibras } \\
\text { obtenidas de dichos tallos. } \\
\text { Tampoco incluye el cáñamo } \\
\text { industrial. (2) Está prohibida la } \\
\text { administración por combustión, } \\
\text { salvo la vaporización. (3) El } \\
\text { limite de suministro máximo } \\
\text { diario es de una onza } \\
\text { equivalente a } 28 \text { gramos de la } \\
\text { flor de Cannabis Medicinal o } \\
\text { su equivalente. Los pacientes } \\
\text { pueden poseer hasta un } \\
\text { máximo de suministro de hasta } \\
30 \text { días. }\end{array}$ & $\begin{array}{l}\text { Solo para condiciones médicas } \\
\text { debilitantes:(1) Alzheimer, (2) } \\
\text { Anorexia, (3) Artritis, (4) Autismo, } \\
\text { (5) Cáncer y el tratamiento de } \\
\text { quimioterapia para el Cáncer, } \\
\text { (6) Depresión, (7) Desórdenes } \\
\text { de Ansiedad, (8) Desórdenes } \\
\text { relacionados al VIH, (9) } \\
\text { Enfermedades degenerativas como } \\
\text { ELAy la esclerosis múltiple, (10) } \\
\text { Enfermedad inflamatoria intestinal, } \\
\text { (11) Enfermedades incurables } \\
\text { y avanzadas que requieran un } \\
\text { cuidado paliativo, (12) Epilepsia, } \\
\text { (13) Fibromialgia, (14) Glaucoma, } \\
\text { (15) Hepatitis C, (16) Insomnio, (17) } \\
\text { Lesiones en el Cordón Espinal, } \\
\text { (18) Migraña, (19) Neuropatías } \\
\text { periferales, (20) Parkinson, (21) } \\
\text { Síndrome de estrés postraumático, } \\
\text { (22) SIDA, (23) Trastorno bipolar, } \\
\text { (24) Cualquier otra condición que } \\
\text { cause caquexia, dolor crónico, } \\
\text { náuseas severas o espasmos } \\
\text { musculares persistentes o que el } \\
\text { Cuerpo Asesor Médico recomiende } \\
\text { y la Junta así lo exprese mediante } \\
\text { Determinación Administrativa. }\end{array}$ \\
\hline Perú & 2017 & $\begin{array}{l}\text { Ley que regula el uso } \\
\text { medicinal y terapéutico } \\
\text { del cannabis y sus } \\
\text { derivados, } 2017 \text { y su } \\
\text { reglamento aprobado } \\
\text { por Decreto Supremo } \\
\mathrm{N}^{\circ} 005-2019-S A \text {. }\end{array}$ & $\begin{array}{l}\text { El paciente debe: (1) } \\
\text { estar registrado en el } \\
\text { Registro Nacional de } \\
\text { Pacientes usuarios } \\
\text { del Cannabis y sus } \\
\text { derivados para uso } \\
\text { medicinal y terapéu- } \\
\text { tico; y (2) contar con } \\
\text { una prescripción } \\
\text { médica consignada } \\
\text { en un recetario } \\
\text { especial. }\end{array}$ & (1) Médico cirujano colegiado & $\begin{array}{l}\text { (1) Se consideran derivados de } \\
\text { Cannabis para uso medicinal } \\
\text { a los medicamentos herbarios, } \\
\text { preparados farmacéuticos, } \\
\text { productos farmacéuticos } \\
\text { y productos naturales de } \\
\text { Cannabis. (2) Se excluye el } \\
\text { uso combustionado o fumado } \\
\text { del Cannabis. (3) La norma no } \\
\text { menciona la cantidad máxima } \\
\text { de posesión y/o suministro. }\end{array}$ & $\begin{array}{l}\text { La norma hace referencia al uso del } \\
\text { cannabis medicinal como "terapia } \\
\text { paliativa para algunas enferme- } \\
\text { dades", sin otras especificaciones. }\end{array}$ \\
\hline
\end{tabular}

similares, independientemente del contexto. Se argumenta, por ejemplo, que la despenalización del cannabis con fines medicinales llevará a la autorización de su consumo recreativo, que la autorización del uso de cannabis medicinal envía a la población un mensaje erróneo («la marihuana es sana o buena»), que los pacientes que utilizan cannabis terminarán abusando de esta sustancia, que los riesgos del cannabis medicinal superan sus beneficios, entre otros ${ }^{(3-5)}$.

El presente artículo, sin embargo, se enfoca en una implicancia ética fundamental de la despenalización del uso del cannabis medicinal que no ha recibido la atención debida durante el debate en el Perú: la relación médico-paciente. Así, se considerarán los desafíos que el cannabis medicinal generan en el ejercicio profesional de la medicina y se desarrollarán tres consideraciones que deberían incorporarse en la normativa peruana con el ánimo de orientar la práctica clínica de los médicos dispuestos a recetar cannabis medicinal. Cabe precisar además que en el presente artículo el término «cannabis medicinal» hace referencia a los derivados del Cannabis para uso medicinal. Es decir, a los medicamentos herbarios, preparados farmacéuticos, productos farmacéuticos y productos naturales de uso en salud derivados de Cannabis; excluyéndose el uso combustionado o fumado del Cannabis, tal como lo establece el Reglamento de la Ley $30681^{(6)}$.

\section{LA RELACIÓN MÉDICO-PACIENTE Y EL CANNABIS MEDICINAL}

Para comprender la naturaleza de la relación médicopaciente es necesario remontarse a la medicina griega y, en especial, a Hipócrates. El juramento hipocrático es por excelencia la referencia de las obligaciones morales de los médicos. A pesar de que los postulados éticos de este juramento sean hoy insuficientes para afrontar los dilemas que la medicina moderna plantea (las técnicas de reproducción asistida, los ensayos clínicos, la eutanasia, etc.), y que su espíritu paternalista haya sido desplazado por el reconocimiento de la autonomía del paciente, los estudiantes de medicina siguen asumiendo el juramento hipocrático (o, al menos, una versión moderna de este) al 
unirse a la profesión. Así pues, la obligación de promover el bien del paciente y de no dañarlo - el principio de beneficencia - que la escuela hipocrática consagró entre los siglos $\mathrm{V}$ y IV a.C. continúa siendo un principio ético vigente hasta la fecha ${ }^{(7)}$.

El principio de beneficencia obliga a los médicos a actuar en atención a los intereses y beneficios de sus pacientes y a minimizar los riesgos ${ }^{(8)}$. En la práctica clínica, ello implica que la principal tarea del médico es brindar a cada uno de sus pacientes la asistencia y los cuidados necesarios, fundados en el conocimiento científico disponible, para la prevención, diagnóstico, tratamiento y rehabilitación de enfermedades ${ }^{(9)}$. En este contexto, el uso del cannabis medicinal les plantea a los médicos importantes desafíos profesionales.

Despenalizado el cannabis medicinal, los médicos deberán juzgar cuándo su prescripción es apropiada. Sin embargo, dicha determinación es problemática y puede llevar a que los médicos eviten prescribir cannabis medicinal o a que lo sobre-prescriban, afectándose en cualquiera de ambos escenarios el bienestar y la salud de los pacientes. La experiencia estadounidense ilustra muy bien esta situación. Por ejemplo, en el estado de Florida, menos del $1 \%$ (484) de médicos habilitados se encuentra registrado para prescribir cannabis ${ }^{(10-11)}$. De la misma manera, en Maryland, sólo el 1\% (172) de médicos está dispuesto a recomendar cannabis ${ }^{(12)}$. Evidencia similar existe sobre los médicos de Nueva York ${ }^{(13)}$. Por el contrario, en Colorado, 15 médicos prescriben cannabis medicinal al $49 \%$ del total de los pacientes que utilizan esta sustancia ${ }^{(14)}$. En Oregon, 1723 médicos prescriben cannabis medicinal y 21 de ellos le recetan al $73 \%$ de los pacientes usuarios registrados ${ }^{(15)}$.

Las razones para no prescribir o sobre-prescribir cannabis medicinal pueden ser diversas (negligencia profesional, conflicto de intereses, etc.). No obstante, una de las razones principales está ligada al hecho de que recomendar el uso del cannabis medicinal no es igual a recomendar cualquier otro tratamiento médico estándar. A diferencia de lo que ocurre con otros tratamientos, la evidencia científica sobre el cannabis medicinal no es aún concluyente para muchos casos. Y, aunque existe limitada información que demuestra la efectividad del cannabis medicinal y casos personales que auto-reportan ciertos beneficios terapéuticos, no se han realizado suficientes ensayos clínicos que garanticen la seguridad y eficacia del cannabis medicinal ${ }^{(16,17)}$

Los ensayos clínicos son parte importante de la investigación en salud y permiten determinar si una nueva intervención es segura y efectiva para la prevención, diagnóstico, tratamiento o curación de enfermedades ${ }^{(18)}$. Sus hallazgos son el sustento científico que los médicos requieren para indicarle a sus pacientes los medicamentos y/o intervenciones apropiadas para su condición de salud. A pesar de que existen algunos estudios que han evaluado distintos cannabinoides y derivados sintéticos o semisintéticos; y otras pocas investigaciones sobre el cannabis herbal, la evidencia de la efectividad del uso del cannabis medicinal es aún limitada; lo que también se ve reflejado en la ausencia de guías clínicas uniformes basadas en la evidencia ${ }^{(16)}$.

En efecto, el informe de The National Academies of Sciences, Engineering and Medicine de los Estados Unidos concluye que la evidencia del uso de cannabinoides orales es contundente para evitar las náuseas producidas por la quimioterapia, para controlar la espasticidad asociada a la esclerosis múltiple y para el tratamiento del dolor crónico en adultos. Sin embargo, para otras indicaciones médicas, la evidencia sobre el cannabis medicinal aún no es robusta y depende de la condición de salud del paciente, de la vía de administración, de la falta de estandarización en las metodologías de investigación, etc. ${ }^{(16,17)}$. Actualmente, cuatro productos farmacéuticos (Marinol, Syndros, Cesamet y Epidiolex) han sido aprobados por la Food and Drug Administration (FDA) de los Estados Unidos. En el caso de los tres primeros, los componentes activos son derivados del cannabis (dronabinol y nabilone). Epidiolex, por su parte, es el primer medicamento compuesto por cannabidiol purificado. Adicionalmente, Sativex continúa siendo investigado. Nabiximols, el ingrediente activo de Sativex, ya ha sido aprobado en otros 15 países: Canadá, España, Alemania, Reino Unido, entre otros ${ }^{(17,19) .}$

Visto este panorama, resulta evidente que la despenalización del uso del cannabis medicinal incrementa considerablemente la responsabilidad de los médicos de velar por los intereses y bienestar de sus pacientes, y de no causarles daño. Aunado a esto, el temor a las represalias legales, los prejuicios y la desinformación dentro de la comunidad médica pueden influir en la decisión de los médicos de no prescribir cannabis. Por ello, es importante que en el protocolo de tratamiento médico que según la Ley $N^{\circ} 30681$, el Ministerio de Salud debe elaborar, se establezcan condiciones claras y uniformes que puedan guiar la decisión de los médicos de recetar cannabis medicinal a sus pacientes; tales como las que se desarrollan a continuación.

\section{REGISTRO Y EDUCACIÓN DE LOS MÉDICOS}

No todos los médicos deben estar obligados a prescribir cannabis medicinal. Tal como ocurre en cualquier otro caso, un médico no debe indicar cierto tratamiento o intervención si considera que esta no es clínicamente apropiada. En ese sentido, un registro que autorice a los médicos a prescribir cannabis medicinal a sus pacientes debe ser implementado. Este registro facilitaría al público saber quiénes están dispuestos a recetar cannabis, ayudaría a generar datos sobre el uso de cannabis medicinal y, sobre todo, garantizaría a los pacientes que sus médicos tratantes cuentan con la formación necesaria para recetarles cannabis medicinal. 
Dado que el cannabis medicinal no es un tratamiento estándar, que su prescripción nunca ha estado legalmente autorizada en la práctica clínica del Perú y que en las Facultades de Medicina no se suelen dictar cursos sobre el uso terapéutico del cannabis, existe naturalmente un gran desconocimiento sobre el cannabis medicinal entre los profesionales de la salud. Por ello, si los médicos deciden prescribir cannabis medicinal, tienen que saber sus componentes y mecanismos de acción, sus efectos, riesgos, vías de administración, manejo de dosis, etc. Ningún médico receta medicamentos (o, al menos, no debería recetar) si es que no conoce la literatura científica correspondiente o si no son parte de su práctica clínica o competencia. Con el cannabis medicinal debe suceder lo mismo.

En este sentido, es importante que, para ser registrado como médico habilitado para prescribir cannabis medicinal, se haya recibido un entrenamiento clínico adecuado y oportuno sobre la materia. Este requisito ha sido, por ejemplo, contemplado por Puerto Rico ${ }^{(20)}$ y los estados de Florida ${ }^{(10)}$, Nueva York ${ }^{(21)}$, Pennsylvania ${ }^{(22)}$, entre otros. Además, (y al menos al inicio) es conveniente que estos programas de educación y registro sean principalmente dirigidos a los médicos que lidian con pacientes potencialmente beneficiarios del cannabis medicinal como, por ejemplo, los oncólogos, los neurólogos o los especialistas en medicina paliativa.

\section{CRITERIOS GENERALES QUE HABILITAN LA PRESCRIPCIÓN DEL CANNABIS}

Otra consideración importante es la relativa a las circunstancias que justifican la prescripción del cannabis. Algunas legislaciones ${ }^{(15,20,23)}$ enumeran las condiciones de salud y/o enfermedades que habilitan al paciente ser usuario del cannabis medicinal. No se discutirá este punto pues la determinación de dichas indicaciones terapéuticas debe obedecer a criterios estrictamente científicos que exceden el ámbito del presente artículo. Lo que resulta interesante destacar es que, aún si existe - o no - una lista de condiciones y/o enfermedades que permitan al paciente ser un usuario del cannabis medicinal, es necesario también adoptar criterios generales con el fin de minimizar los posibles daños y riesgos del cannabis medicinal.

Por ejemplo, se podría establecer que el cannabis medicinal sea prescrito sólo si otras opciones de tratamiento estándar no han sido efectivas o si los efectos secundarios de los tratamientos convencionales son intolerables para el paciente ${ }^{(14,24)}$. Así, por ejemplo, lo recomiendan la Oficina del Cannabis Medicinal de Holanda (25) y los colegios profesionales de médicos y cirujanos de las provincias canadienses de Alberta ${ }^{(26)}$, British Columbia ${ }^{(27)}$, Newfoundland and Labrador (28), Prince Edward Island (29), etc. Además, algunos otros criterios generales que podrían ser útiles para calificar a un paciente como beneficiario del cannabis medicinal que la experiencia internacional ha tomado en cuenta son: a) que el paciente no tenga ni haya tenido un historial de abuso y adicción a las drogas, b) que no esté embarazada, c) que no se encuentre durante el periodo de lactancia, d) que no presente trastornos psicóticos ni tenga una historia familiar de estos, entre otros ${ }^{(27,28,30)}$.

Definitivamente estos criterios generales que habilitarían la prescripción del cannabis medicinal no son las únicos y la aplicación de estos no siempre será obligatoria. Los médicos tendrán que evaluar, paciente por paciente, en atención a los riegos y beneficios, si el cannabis es el mejor curso de tratamiento, y estos criterios generales no son más que una guía para tomar y justificar sus decisiones.

\section{UNA RELACIÓN BONA-FIDE}

Finalmente, es recomendable que el cannabis medicinal sólo se recete por médicos registrados, en el marco de una relación médico-paciente bona-fide (buena fe). Es decir, cuando la relación entre el médico tratante y el paciente sea una relación establecida en el tiempo, en donde el médico le presta continuamente servicios de salud y es el primer responsable de su diagnóstico, cuidado y tratamiento. De esta manera, el médico debe llevar a cabo todos los exámenes necesarios y conocer con precisión la historia clínica de su paciente antes de decidir si el cannabis medicinal es la mejor opción. Una vez prescrito el cannabis, el paciente debe ser periódicamente evaluado para determinar si el cannabis beneficia o no su estado de salud.

La indicación de cualquier tratamiento o intervención médica tiene como base el diagnóstico del paciente. Al no ser el cannabis medicinal una práctica clínica estándar aún, los médicos que lo prescriben tienen una mayor discrecionalidad y responsabilidad y, por lo tanto, deben ser más cuidadosos con la evaluación del paciente, su diagnóstico y el plan de acción que decidan indicar. Una relación bona-fide entre el médico que prescribe cannabis y su paciente facilita y promueve este deber "reforzado» de cuidado y protege legal y moralmente al médico tratante pues su decisión habrá sido estrictamente evaluada y monitoreada. Una relación bona-fide puede evitar además que los pacientes recorran distintos consultorios médicos con el ánimo de encontrar algún doctor dispuesto a autorizarles el uso del cannabis sin mayor evaluación.

La necesidad de una relación bona-fide entre el médico y su paciente para la prescripción de cannabis medicinal ha sido adoptada por la Federación de Colegios Profesionales de los Estados Unidos (Federation of State Medical Boards) ${ }^{(31)}$. Asimismo, este requisito ha sido considerado por Puerto Rico ${ }^{(20)}$ y los estados estadounidenses de Colorado ${ }^{(14)}$, Nueva York ${ }^{(21,23)}$, el distrito de Columbia ${ }^{(32)}$, etc. Los colegios profesionales de médicos y cirujanos de algunas provincias de Canadá como, por ejemplo, British Columbia ${ }^{(27)}$, Nova Scotia ${ }^{(33)}$ y New Brunswick ${ }^{(34)}$ han también 
incorporado el criterio de la relación médico-paciente bona-fide para la prescripción de marihuana medicinal.

\section{CONCLUSIONES}

Dado el status quo del cannabis medicinal y sus implicancias éticas en la relación médico-paciente, es importante que la normativa peruana y, en concreto, el protocolo de tratamiento médico a cargo del Ministerio de Salud adopte condiciones que garanticen que aquellos pacientes que requieran el cannabis medicinal realmente accedan a este, sin que ello comprometa el cumplimiento de las obligaciones morales de los médicos en el ejercicio de su profesión.

El registro y educación de los médicos, la formulación de criterios generales que habilitan al paciente utilizar el cannabis medicinal, así como el requisito de una relación bona-fide entre el médico tratante y su paciente que han sido desarrolladas en el presente artículo son algunas consideraciones bioéticas importantes a tomar en cuenta. Sin embargo, no agotan la discusión pues existen, sin duda, otras cuestiones éticas relevantes para la relación médicopaciente en el marco del uso del cannabis medicinal como el consentimiento informado del paciente, el mal uso o abuso del cannabis medicinal, los conflictos de interés, entre otros.

La tarea no es fácil, pero confiamos en que nuestras autoridades, con la participación del Colegio Médico del Perú, adoptarán disposiciones que consideren el impacto del uso del cannabis medicinal en la relación médico-paciente y permitan a los médicos seguir velando por la salud y bienestar de todos los peruanos.

\section{Fuentes de financiamiento: Autofinanciado}

Conflictos de interés: La autora declara no tener conflictos de intereses en la publicación del artículo.

\section{REFERENCIAS BIBLIOGRÁFICAS}

1. Nathan DL, Clark HW, Elders J. The Physicians' Case for Marijuana Legalization. AJPH. 2017; 7(11): 1746-1747.

2. Ley $\mathrm{N}^{\circ} 30681$, Ley que regula el uso medicinal y terapéutico del cannabis y sus derivados, 17 de noviembre de 2017 (PE)

3. Clark PA. The Ethics of Medical Marijuana: Government Restrictions vs. Medical Necessity. J Public Health Policy. 2000; 21(1): 40-60.

4. Gregorio J. Physicians, Medical Marijuana, and the Law. VM. 2014; 16(9): 732-738.

5. Wilkinson ST, D'Souza DC. Problems with the medicalization of marijuana. JAMA. 2014; 311(23): 2377-2378.

6. Decreto Supremo $N^{\circ}$ 005-2019-SA que aprueba el Reglamento de la Ley $\mathrm{N}^{\circ}$ 30681, Ley que regula el uso medicinal y terapéutico del Cannabis y sus derviados, 25 de febrero de 2019 (PE).

7. DeGrazia D, Mappes TA, Brand-Ballard J. Biomedical Ethics. 7ma Edición. New York: McGraw-Hill; 2011.

8. Estados Unidos. The Belmont Report: Ethical Principles and Guidelines for the Protection of Human Subjects of Research. Bethesda, MD: The Commission, 1978.

9. Levine RJ. Research and Practice. In: Emanuel EJ, Crouch RA, Arras JD, Moreno JD, Grady C, editors. Ethical and Regulatory Aspects of Clinical Research. Baltimore/London: The Johns Hopkins University Press. p. 103-107.
10. Rubin R. Medical Marijuana is Legal in Most States, but Physicians Have Little Evidence to Guide Them. JAMA. 2017; 317(16): 1611-1613.

11. Call J. Marijuana is Available, but the Doctor's not in [Internet]. Tallahassee Democrat. 25 Jul 2016 [citado 22 Nov 2017]. Disponible en: http://www. tallahassee.com/story/news/2016/07/25/ marijuana-available-but-doctorsnot/87543740/

12. Cohn M, McDaniels AK. Few doctors sign on to recommend medical marijuana in Maryland [Internet]. The Baltimore Sun. 14 Nov 2016 [citado 22 Nov 2017]. Disponible en: http://www.baltimoresun. com/news/maryland/bs-hs-marijuanadoctors-20161112-story.html

13. Lewis C. New York doctors just say no to medical marijuana [Internet]. Crain's New York Business. 31 Jul 2015 [citado 22 Nov 2017] Disponible en: http://www. crainsnewyork.com/article/20150728/ HEALTH_CARE/150729843/newyork-doctors-just-say-no-to-medicalmarijuana

14. Nussbaum AM, Boyer JA, Kondrad EC. "But my Doctor Recommend Pot": Medical Marijuana and the PatientPhysician Relationship. J Gen Intern Med. 2011; 26(11): 1364-1367.

15. HB 4014 Clinical Guidelines Work Group. Medical Marijuana Recommendation Guidelines for Physicians [Internet]. Oregon (US): Public Health Division of the Oregon
Health Authority; 2016 [citado 22 Nov 2017] 18 p. Disponible en: http://www. oregon.gov/oha/PH/PreventionWellness/ marijuana/Documents/OHA-9262Attending-Physician-Guidelines.pdf

16. Unidad de Análisis y Generación de Evidencias en Salud Pública del Instituto Nacional de Salud. Actualización de la Revisión y Síntesis de la Evidencia sobre Regulación del Uso Médico de Cannabis [Internet]. Lima (PE): Instituto Nacional de Salud; 2017 [citado 19 Nov 2017] 95 p. Disponible en: http:// www.ins.gob.pe/repositorioaps/0/0/ jer/CNSP/unagesp/publicaciones/ notas_tecnicas/Actualizacion\%20de\%20 la\%20Revision $\% 20 y \% 20$ Sintesis\%20 de\%20la\%20Evidencia\%20sobre\%20 Regulacion\%20del\%20Uso\%20 Medico\%20de\%20Cannabis.pdf

17. The National Academies of Sciences, Engineering and Medicine. The Health Effects of Cannabis and Cannabinoids: the $\mathrm{Cu}$ rrent State of Evidence and Recommendations for Research [Internet]. Washington DC (US): The National Academies Press; 2017 [citado 19 Nov 2017] 468 p. Disponible en: https://www.nap.edu/cata$\log / 24625 /$ the-health-effects-of-cannabisand-cannabinoids-the-current-state

18. Decreto Supremo No 021-2017-SA, Reglamento de Ensayos Clínicos, 30 de junio de 2017 (PE)

19. U.S. Food \& Drug Administration. FDA and Marijuana [Internet]. 2017 [citado 10 
Dic 2018] Disponible en: https://www. fda.gov/NewsEvents/PublicHealthFocus/ ucm 421163.htm

20. Reglamento No 9038, 2 de julio de 2018 (PR)

21. New York State Department of Health. Medical Use of Marijuana Under the Compassionate Care Act: Two-year Report [Internet]. New York; 2017. 13p. [citado 22 Nov 2017] Disponible en: https://www.health.ny.gov/regulations/ medical_marijuana/docs/two_year_ report.pdf

22. Pennsylvania Department of Health. Medical Marijuana Program [Internet]. 2017 [citado 22 Nov 2017] Disponible en: https://www.pa.gov/guides/pennsylvania-medical-marijuana-program/

23. New York State Department of Health. Medical Marijuana Program [Internet]. 2017 [citado 22 Nov 2017] Disponible en: https://www.health.ny.gov/regulations/ medical_marijuana/

24. Voth EA. Guidelines for Prescribing Medical Marijuana. West J Med. 2001; 175(5):305-306.

25. Ministry of Health, WelfareandSport.Office of Medical Cannabis [Internet]. Holanda; 2018 [citado 10 Dic 2018] Disponible en: https://english.cannabisbureau.nl/

26. College of Physicians and Surgeons of Alberta. Standard of Practice: Cannabis for Medical Purposes [Internet]. Alberta;
2014 [citado 22 Nov 2017] Disponible en: http://www.cpsa.ca/standardspractice/ cannabis-for-medical-purposes/

27. College of Physicians and Surgeons of British Columbia. Professional Standards and Guidelines: Cannabis for Medical Purposes [Internet]. British Columbia; 2015 [citado 22 Nov 2017] Disponible en: https://www.cpsbc.ca/files/pdf/PSGCannabis-for-Medical-Purposes.pdf)

28. College of Physicians and Surgeons of Newfoundland and Labrador. Medical Marijuana: Advisory and Interim Guideline [Internet]. Newfoundland and Labrador; 2014 [citado 22 Nov 2017] Disponible en: http://www.cpsnl.ca/WEB/CPSNL/ Policies/Advisory_and_Interim_ Guideline_-_Medical_Marihuana.aspx

29. College of Physicians and Surgeons of Prince Edward Island. Prescribing for Medical Marijuana [Internet]. Prince Edward Island; 2016 [citado 22 Nov 2017] Disponible en: http://cpspei.ca/wp-content/ uploads/2017/03/Marijuana-Prescribing-Nov-3016.pdf

30. (32) College of Physicians and Surgeons of Ontario. Marijuana for Medical Purposes [Internet]. Ontario; 2002 [citado 22 Nov 2017] Disponible en: http://www. cpso.on.ca/policies-publications/policy/ marijuana-for-medical-purposes

31. Federation of State Medical Boards. Model Guidelines for the Recommendation of Marijuana in Patient Care [Internet]. 2016. 14p. [citado 22 Nov 2017] Disponible en: https://www.fsmb.org/Media/Default/ PDF/BRD_RPT_16-2_Marijuana_ Model_Guidelines.pdf

32. Government of the District of Columbia Department of Health. Medical Marijuana Program Physician Frequently Asked Questions [Internet]. 2017 [citado 22 Nov 2017] Disponible en: https://doh. dc.gov/sites/default/files/dc/sites/doh/ publication/attachments/120430FAQPhysicians\%20Final.pdf

33. College of Physicians and Surgeons of Nova Scotia. Professional Standard Regarding the Authorization of Marijuana for Medical Purposes [Internet]. Nova Scotia; 2014 [citado 22 Nov 2017] Disponible en: https://cpsns.ns.ca/wp-content/ uploads/2017/10/Authorization-of-Marijuana-for-Medical-Purposes.pdf

34. College of Physicians and Surgeons of New Brunswick. Medical Marijuana Guidelines [Internet]. New Brunswick; 2014 [citado 22 Nov 2017] Disponible en: http://www. cpsnb.org/en/medical-act-regulationsand-guidelines/guidelines/444-medicalmarijuana

Correspondencia: Sarah Carracedo Dirección: Jr. Hemano Lobo 133, Lima 15037, Perú.

Correo electrónico: sarah.carracedo@pucp.edu.pe 